BULL, AUSTRAL, MATH. SOC.

$26 \mathrm{D} 15,47 \mathrm{~A} 30$

VOL. 34 (1986) 225-232

\title{
A REVERSED HARDY INEQUALITY
}

\author{
P.F, RENAUd
}

We consider Hardy's classical inequality for Cesaro averages and show that a reversed version exists if we restrict ourselves to monotone sequences. Some consequences of this result as well as an integral version are also obtained.

\section{Introduction}

Let $S$ be the Cesaro operator defined on sequences $x=\left(x_{n}\right)_{1}^{\infty}$ by

$$
(S x)_{n}=\frac{1}{n} \sum_{k=1}^{n} x_{k} \quad n \geq 1 .
$$

If $p>1$, a classical inequality due to Hardy (see for example [1] p.239) shows that $S$ is a bounded linear operator on $\ell_{p}$ with

$$
\|S x\|_{p} \leq \frac{p}{p-1}\|x\|_{p}
$$

Now the operator $S$ is one-to-one but not onto and hence has no bounded inverse. In other words no inequality of the form

$$
\|S x\|_{p} \geq K_{p}|| x \mid \|_{p} \quad\left(K_{p} \quad \text { independent of } x\right)
$$

can be universally true in $\ell_{p}$.

On the other hand we will show that such an inequality does hold if

Received 13 November 1985.

Copyright Clearance Centre, Inc. Serial-fee code: 0004-9727/86 $\$ A 2.00+0.00$. 
we restrict ourselves to monotone sequences. Specifically we have the following:

THEOREM 1. If $p>1$ and $x=\left(x_{n}\right)_{1}^{\infty} \in \ell_{p}$ with $x_{1} \geq x_{2} \geq \ldots \geq 0$, then (writing $\|\cdot\|$ for $\|\cdot\| \|_{p}$ )

$$
\|s x\|^{p} \geq \zeta(p)\|x\|^{p}
$$

where $\zeta$ is the Riemann Zeta function.

The constont $\zeta(p)$ is best possible.

This generalizes a recent result of Lyons [2] who proved the case $p=2$. It could also be noted that the resulting inequality

$$
\zeta(p) \leq\left(\frac{p}{p-1}\right)^{p}
$$

is of course at its tightest when $p$ is close to 1 .

\section{Proof of the Inequality.}

We begin with the following simple lema.

LEMMA 1. For $p>1$ and $x_{1} \geq x_{2} \geq \ldots \geq x_{n} \geq 0$,

$$
\left(x_{1}+\ldots+x_{n}\right)^{p}-\left(x_{1}^{p}+\ldots+x_{n}^{p}\right) \geq \sum_{k=2}^{n}\left(k^{p}-(k-1)^{p}-1\right) x_{k}^{p} \text {. }
$$

Proof. Fix $k$ and write $u=x_{1}+\ldots+x_{k-1}$ and $x=x_{k}$ so that $u \geq(k-1) x$. Then

$$
\begin{aligned}
\left(x_{1}+\ldots+x_{k}\right)^{p}-\left(x_{1}+\ldots+x_{k-1}\right)^{p} & =(u+x)^{p}-u^{p} \\
& =x^{p}\left[(\alpha+1)^{p}-\alpha^{p}\right]
\end{aligned}
$$

where $\alpha=\frac{u}{x} \geq k-1$.

Now the function $f(x)=(x+1)^{p}-x^{p}$ is increasing so that $f(u / x) \geq f(k-1)$. It follows that

$$
\begin{aligned}
\left(x_{1}+\ldots+x_{k}\right)^{p}-\left(x_{1}+\ldots+x_{k-1}\right)^{p} & =x^{p} f(u / x) \\
& \geq x^{p} f(k-1),
\end{aligned}
$$

that is

$$
\left(x_{1}+\ldots+x_{k}\right)^{p}-\left(x_{1}+\ldots+x_{k-1}\right)^{p} \geq\left(k^{p}-(k-1)^{p}\right) x_{k}^{p} .
$$


Sumning this inequality from $k=2$ to $k=n$ yields

$$
\left(x_{1}+\ldots+x_{n}\right)^{p}-x_{1}^{p} \geq \sum_{k=2}^{n}\left(k^{p}-(k-1)^{p}\right) x_{k}^{p}
$$

from which (2) follows immediately.

Now let $x=\left(x_{n}\right) \in \ell_{p}$ with $x_{1} \geq x_{2} \geq \ldots \geq 0$. Then

$$
\begin{aligned}
\|S x\|^{p} & =\sum_{n=1}^{\infty} \frac{\left(x_{1}+\ldots+x_{n}\right)^{p}}{n^{p}} \\
& =\sum_{n=1}^{\infty} \frac{\left(x_{1}^{p}+\ldots+x_{n}^{p}+\varepsilon_{n}\right)}{n^{p}},
\end{aligned}
$$

where $\varepsilon_{1}=0$ and, for $n \geq 2, \varepsilon_{n}=\left(x_{1}+\ldots+x_{n}\right)^{p}-\left(x_{1}^{p}+\ldots+x_{n}^{p}\right)$ so that by Lemma 1 ,

$$
\varepsilon_{n} \geq \sum_{k=2}^{n}\left(k^{p}-(k-1)^{p}-1\right) x_{k}^{p}
$$

Hence $\| S x||^{p}=\sum_{n=1}^{\infty}\left(\frac{1}{n}+\frac{1}{(n+1)^{p}}+\cdots\right) x_{n}^{p}+\sum_{n=2}^{\infty} \frac{\varepsilon_{n}}{n^{p}}$

or

$$
\|S x\|^{p}=\zeta(p)\|x\|^{p}+\sum_{n=2}^{\infty}\left[\frac{\varepsilon_{n}}{n^{p}}-\left(\sum_{k=1}^{n-1} \frac{1}{k^{p}}\right) x_{n}^{p}\right] .
$$

But from (4), $\sum_{n=2}^{\infty} \frac{\varepsilon_{n}}{n^{p}} \geq \sum_{n=2}^{\infty} \frac{1}{n^{p}} \sum_{k=2}^{n}\left(k^{p}-(k-1)^{p}-1\right) x_{k}^{p}$

$$
=\sum_{n=2}^{\infty}\left(n^{p}-(n-1)^{p}-1\right)\left(\frac{1}{n^{p}}+\frac{1}{(n+1)^{p}}+\ldots\right) x_{n}^{p} .
$$

Now let $S_{n}=\sum_{k=1}^{n} \frac{1}{k^{p}}$ and $T_{n}=\zeta(p)-S_{n}=\sum_{k=n+1}^{\infty} \frac{1}{k^{p}}$, so that we may write (6) as

$$
\sum_{n=2}^{\infty} \frac{\varepsilon_{n}}{n^{p}} \geq \sum_{n=2}^{\infty}\left(n^{p}-(n-1)^{p}-1\right) T_{n-1^{x}}^{p}
$$

From (5), Theorem 1 will follow once we show that 


$$
\sum_{n=2}^{\infty}\left(\frac{\varepsilon_{n}}{n^{p}}-\sum_{k=1}^{n-1} \frac{x^{p}}{k^{p}}\right) \geq 0
$$

and from (7) it suffices to show:

\section{LEMMA 2. For $n \geq 2$,}

$$
\left(n^{p}-(n-1)^{p}-1\right) T_{n-1} \geq S_{n-1} \text {. }
$$

Before proceeding to the proof, it is interesting to note in passing that the case $n=2$ amounts to the usual comparison between $\sum \frac{1}{n^{p}}$ with a geometric series.

We are required to show that

$$
\left(2^{p}-2\right)(\zeta(p)-1) \geq 1 \text {, }
$$

that is that

$$
\zeta(p) \geq 1+\frac{1}{2^{p}-2}
$$

$$
\text { But } \begin{aligned}
\zeta(p) & =1+\frac{1}{2^{p}}+\frac{1}{3^{p}}+\frac{1}{4^{p}}+\ldots \\
& \geq 1+\frac{1}{2^{p}}+\frac{2}{4^{p}}+\ldots \\
& =1+\frac{1}{2^{p}}\left(1+\frac{1}{2^{p-1}}+\frac{1}{2^{2(p-1)}}+\cdots\right)=1+\frac{1}{2^{p}-2} .
\end{aligned}
$$

Proof of Lemma 2. Fix $n \geq 2$. We have

$$
\left(n^{p}-(n-1)^{p}-1\right) T_{n-1}=\sum_{k=0}^{\infty}\left(\frac{n}{n+k}\right)^{p}-\sum_{k=n}^{\infty} \frac{(n-1)^{p}}{k^{p}}-\sum_{k=n}^{\infty} \frac{1}{k^{p}} .
$$

In the right hand side of (9), the terms in the first sum corresponding to $k=n^{2}-n, n^{2}, n^{2}+n$ etcetera, cancel with the terms in the third sum corresponding to $k=n, n+1, n+2$ etcetera, respectively. So we have

$$
\begin{aligned}
&\left(n^{p}-(n-1)^{p}-1\right) T_{n-1}= \sum_{k=0}^{n^{2}-n-1}\left(\frac{n}{n+k}\right)^{p} \\
&+\sum_{j=0}^{\infty} \sum_{k=1}^{n-1}\left(\frac{n}{n^{2}+j n+k}\right)^{p} \\
&-\sum_{k=n}^{\infty} \frac{(n-1)^{p}}{k^{p}}
\end{aligned}
$$




$$
\begin{aligned}
= & \sum_{k=0}^{n^{2}-n-1}\left(\frac{n}{n+k}\right)^{p}-\sum_{k=n}^{n^{2}-n}\left(\frac{n-1}{k}\right)^{p} \\
& +\sum_{j=0}^{\infty} \sum_{k=1}^{n-1}\left[\left(\frac{n}{n^{2}+j n+k}\right)^{p}-\left(\frac{n-1}{n^{2}+(j-1) n-j+k}\right)^{p}\right]
\end{aligned}
$$

But the term in the double sum is positive so that (10) becomes

$$
\left(n^{p}-(n-1)^{p}-1\right) T_{n-1} \geq \sum_{k=0}^{n^{2}-n-1}\left(\frac{n}{n+k}\right)^{p}-\sum_{k=n}^{n^{2}-n}\left(\frac{n-1}{k}\right)^{p}
$$

Now remove the terms corresponding to $k=0, n, 2 n \ldots(n-2) n$ from the first sum. The remaining $(n-1)^{2}$ terms can be grouped with the $(n-1)^{2}$ terms of the second sum to obtain a square array so that (11) becomes

$$
\left(n^{p}-(n-1)^{p}-1\right) T_{n-1} \geq \sum_{k=1}^{n-1} \frac{1}{k^{p}}+\sum_{j=1}^{n-1} \sum_{k=1}^{n-1}\left[\left(\frac{n}{j n+k}\right)^{p}-\left(\frac{n-1}{j(n-1)+k}\right)^{p}\right]
$$

and since $\frac{n}{j n+k} \geq \frac{n-1}{j(n-1)+k}$ we obtain

$$
\left(n^{p}-(n-1)^{p}-1\right) T_{n-1} \geq S_{n-1}
$$

which proves Lemma 2 and hence Theorem 1 . That $\zeta(p)$ is the best possible constant can be seen from considering the case when $x_{2}=x_{3}=\ldots=0$.

A large number of inequalities have been derived from Hardy's inequality. It would seem that most of these have reversed versions. One interesting aspect is the disparity in levels of difficulty one encounters in the proofs. As an example, the reversed version of Hilbert's inequality seems quite non-trivial and will be discussed elsewhere. On the other hand, Carleman's reversed inequality is entirely trivial. Somewhere in between, usually based on Lemma 1, fall examples corresponding to inequalities such as Hardy's dual inequality ([1] p. 246).

$$
\text { If } p>1 \text {, then } \Sigma\left(x_{n}+x_{n+1}+\ldots\right)^{p} \leq p^{p} \sum\left(n x_{n}\right)^{p} \text {. }
$$

The reversed version is

THEOREM 2. If $p>1, x_{1} \geq x_{2} \geq \ldots \geq 0$ then 


$$
\sum_{n=1}^{\infty}\left(x_{n}+x_{n+1}+\ldots\right)^{p} \geq \sum_{n=1}^{\infty}\left(n x_{n}\right)^{p}
$$

Proof. From Lemma 1 we have

$$
\left(x_{1}+\ldots+x_{n}\right)^{p} \geq \sum_{k=1}^{n}\left(k^{p}-(k-1)^{p}\right) x_{k}^{p}
$$

so that

$$
\begin{aligned}
\sum_{n=1}^{\infty}\left(x_{n}+x_{n+1}+\ldots\right)^{p} & \geq \sum_{n=1}^{\infty} \sum_{k=1}^{\infty}\left(k^{p}-(k-1)^{p}\right) x^{p} n+k-1 \\
& =\sum_{n=1}^{\infty}\left(n x_{n}\right)^{p} .
\end{aligned}
$$

\section{Reversed Inequalities for Integrals.}

The corresponding Hardy inequality for integrals is given by

$$
\int_{0}^{\infty}\left[\frac{F(x)}{x}\right]^{p} d x \leq\left(\frac{p}{p-1}\right)^{p} \int_{0}^{\infty}[f(x)]^{p} d x
$$

where $p>1, f(x) \geq 0$ and $F(x)=\int_{0}^{x} f(t) d t$.

As may be expected, a reversed version of (13) exists. It should be noted that the constants differ in the sum and integral case whereas for the classical inequalities they are the same. specifically we have

THEOREM 3. Let $f \in L_{p}[0, \infty), f+0, p>1$ and put $F(x)=\int_{0}^{x} f(t) d t$. Then

$$
\int_{0}^{\infty}\left[\frac{E(x)}{x}\right]^{p} d x \geq \frac{p}{p-1} \int_{0}^{\infty}[f(x)]^{p} d x
$$

(Alternatively to highlight the similarity with Theorem 1

$$
\left.\|S f\|^{p} \geq \frac{p}{p-1}\|f\|^{p} \text { where } S f(x)=\frac{F(x)}{x} .\right)
$$

Proof. For $x \geq 0, F(x)=\int_{0}^{x} f(t) d t \geq x f(x)$ so that

$$
p f(x)[F(x)]^{p-1} \geq p x^{p-1}[f(x)]^{p}
$$


A Reversed Hardy Inequality

231

which yields by integration

$$
[F(x)]^{p} \geq p \int_{0}^{x} t^{p-1}[f(t)]^{p} d t \quad \text { almost everywhere. }
$$

(The fact that ${ }_{F}^{p}$ is differentiable almost everywhere and has derivative p $f F^{p-1}$ follows from standard results in measure theory once we assume that $f$ is monotone.)

So

$$
\begin{aligned}
\|S f\|^{p} & =\int_{0}^{\infty}\left[\frac{E(x)}{x}\right]^{p} d x \\
& \geq \int_{0}^{\infty} \frac{p}{x^{p}} \int_{0}^{x} t^{p-1}[f(t)]^{p} d t d x \\
& =\int_{0}^{\infty}\left[\int_{t}^{\infty} \frac{p t^{p-1}[f(t)]^{p}}{x^{p}} d x\right] d t
\end{aligned}
$$

(by Fubini's Theorem),

$$
=\frac{p}{p-1} \int_{0}^{\infty}[f(t)]^{p} d t
$$

There is also an integral analogue of Theorem 2 .

THEOREM 4. Let $f \in L_{p}[0, \infty), f+0, p>1$. Then

$$
\int_{0}^{\infty}\left[\int_{x}^{\infty} f(t) d t\right]^{p} d x \geq \int_{0}^{\infty}[x f(x)]^{p} d x
$$

Proof. For $x, y \geq 0$ we have

$$
\int_{x}^{x+y} f(t) d t \geq y f(x+y),
$$

so that

$$
p\left[\int_{x}^{x+y} f(t) d t\right]^{p-1} f(x+y) \geq p y^{p-1}[f(x+y)]^{p}
$$

and by integration

$$
\left[\int_{x}^{x+y} f(t) d t\right]^{p} \geq p \int_{0}^{y} t^{p-1}[f(x+t)]^{p} d t .
$$

https://doi.org/10.1017/S0004972700010091 Published online by Cambridge University Press 
Letting $y \rightarrow \infty$

$$
\begin{aligned}
{\left[\int_{x}^{\infty} f(t) d t\right]^{p} } & \geq p \int_{0}^{\infty} t^{p-1}[f(x+t)]^{p} d t \\
& =p \int_{x}^{\infty}(u-x)^{p-1}[f(u)]^{p} d u,
\end{aligned}
$$

and hence

$$
\begin{aligned}
\int_{0}^{\infty}\left[\int_{x}^{\infty} f(t) d t\right]^{p} d x & \geq p \int_{0}^{\infty}\left[\int_{x}^{\infty}(u-x)^{p-1}[f(u)]^{p} d u\right] d x \\
& =p \int_{0}^{\infty}\left[\int_{0}^{u}(u-x)^{p-1}[f(u)]^{p} d x\right] d u \\
& =p \int_{0}^{\infty}[f(u)]^{p}\left[\int_{0}^{u}(u-x)^{p-1} d x\right] d u \\
& =\int_{0}^{\infty}[u f(u)]^{p} d u
\end{aligned}
$$

\section{References}

[1] G. H. Hardy, J. E. Littlewood and G. Polya, Inequalities, (Cambridge University Press, London, New York 1964).

[2] R. Lyons, "A lower bound on the Cesaro operator", Proc. Amer. Math. Soc. 86 (1982), 694.

\footnotetext{
Department of Mathematics,

University of Canterbury

Private Bag

Christchurch

New Zealand.
} 